\title{
SPECTRA OF PERIODIC NONRECIPROCAL ELECTRIC CIRCUITS*
}

\author{
ALEX FIGOTIN $^{\dagger}$ AND ILYA VITEBSKY ${ }^{\dagger}$
}

\begin{abstract}
Spectral properties of periodic nonreciprocal (gyrotropic) electric circuits incorporating gyrators are studied. Based on the Lagrange formalism we analyze the origin of the special features of periodic nonreciprocal media and establish some universal spectral properties common for a variety of gyrotropic periodic systems. We found that the combination of periodicity and nonreciprocity can produce asymmetric dispersion relations implying a significant difference in the wave energy transfer in opposite directions.
\end{abstract}

Key words. nonreciprocal circuits, spectra, gyrotropic medium, periodic medium, gyrator, photonic crystals

AMS subject classifications. 35B27, 65F $15,73 \mathrm{D} 25,78 \mathrm{~A} 45$

PII. S0036139900370583

1. Introduction. We study in this paper the spectral properties of nonreciprocal periodic media (for basic definitions of nonreciprocal materials see [8, Chapter 15], $[10$, sections 1.1, 2.1]). Here are several important examples of such media: (i) one-, two-, and three-dimensional photonic crystals, i.e., periodic dielectric structures with at least one dielectric component being a nonreciprocal dielectric material with some kind of magnetic order; (ii) periodic electric networks containing so-called gyrators [15], [4, section 5.1], which are basic nonreciprocal network elements; (iii) periodic microwave networks incorporating nonreciprocal devices based on the gyromagnetic effect; for instance, Faraday rotation insulators, gyrators, four-port rotation circulators, nonreciprocal Faraday rotation-type phase shifters, nonreciprocal directional couplers and more $[8$, Chapters $15,16,17]$. A particular focus of our studies is on the interplay between the periodicity and nonreciprocity. One of the main effects of this interplay is the difference between group velocities for the waves propagating in opposite directions.

We consider infinite periodic electric meshes composed of interconnected linear lossless passive elements. Such a network can always be represented as a periodic array of four basic network elements: capacitors, inductors, transformers, and gyrators (nonreciprocal elements). An arbitrary network can be analyzed using some welldeveloped methods of network analysis (see, for instance, [4]). Our interest in electric circuits is twofold. First, lumped nonreciprocal periodic circuits can be used in many practical applications including direction discrimination. These applications are normally limited to a low-frequency range. Second, nonreciprocal circuits are among the simplest examples of nonreciprocal wave conducting media and as such they give valuable insights to more complicated periodic systems, for instance, photonic crystals.

${ }^{*}$ Received by the editors March 31, 2000; accepted for publication (in revised form) December 6, 2000; published electronically April 24, 2001. This research was sponsored by the Air Force Office of Scientific Research, Air Force Materials Command, USAF, under grant F49620-99-1-0203. The U.S. Government is authorized to reproduce and distribute reprints for governmental purposes notwithstanding any copyright notation thereon. The views and conclusions contained herein are those of the authors and should not be interpreted as necessarily representing the official policies or endorsements, either expressed or implied, of the Air Force Office of Scientific Research or the U.S. Government.

http://www.siam.org/journals/siap/61-6/37058.html

${ }^{\dagger}$ Department of Mathematics, University of California at Irvine, Irvine, CA 92697-3875 (afigotin@ math.uci.edu,dvitebsk@math.uci.edu). 
Therefore, periodic electric meshes can be used for computer and physical simulation of electromagnetic phenomena in photonic crystals and other complex periodic systems.

Though the primary focus of this paper is on electric nonreciprocal networks, we would like to keep in sight other nonreciprocal periodic systems. There is a wellestablished analogy between the theory of electric networks and the electrodynamics of continuous dielectric media; see, for instance, [7, Chapter 1]. Relative simplicity of the theory of electric networks allows us to study analytically many fundamental phenomena that cannot be addressed in the electrodynamics without recourse to some questionable approximations. On the other hand, many theoretical concepts and techniques first developed for linear excitations in crystalline materials can be carried over to periodic electric networks. In particular, some important qualitative spectral features of nonreciprocal periodic networks can be predicted and analyzed using rigorous symmetry arguments borrowed from the theory of magnetically ordered crystals such as ferro- and antiferromagnets. So obtained results hold regardless of the degree of network complexity. In this paper we will not explore such ideas.

Turning to the reciprocity concept, we recall that in its simplest sense, the reciprocity means that a response of a system to a source is unchanged when the source and the measurer are interchanged. In a more general sense, reciprocity theorems relate a response at one location due to a source at another location to the response at the second location due to a source at the first location. For dielectric media the relevant statement is the Lorentz reciprocity theorem [7, Chapters 3-8], [3, Appendix F.2]. The reciprocity in the electric circuit theory reduces to the symmetry of the impedance matrix $Z=\left\{Z_{i j}\right\}$ [7, Chapters 3-8], [5, section 2.3]. Respectively, for nonreciprocal circuits (i.e., for those incorporating gyrators) matrix $Z$ is generally asymmetric. For an insightful view of the origins of the properties of nonreciprocal (gyrotropic) media see also [5, section 8].

One of most important results in this paper is that a combination of periodicity and nonreciprocity can cause qualitative alterations of the dispersion relations $\Omega(\vec{k})$. Graphic examples of such a "skewed" (asymmetric) dispersion law are shown in Figures 3.4 and 3.5. The asymmetry of the dispersion relations implies that nonreciprocal circuits can exhibit a significant difference in the wave energy transfer in opposite directions.

The paper is organized as follows. In section 2 we introduce the concept of spectral nonreciprocity. This fundamental spectral feature results from nonreciprocity of the linear response (e.g., the property of $Z_{i j} \neq Z_{j i}$ ) combined with spatial periodicity of the system. In sections 3 and 4 we outline some basic properties and phenomena stemming from the combination of nonreciprocity and periodicity. We give a concise exposition of the basic phenomena for a typical electric network such as shown in Figure 3.3. In the following sections 5 and 6 we prove the basic properties of nonreciprocal periodic networks. When approaching these problems we employ the Lagrange formalism [6], [13], [14]. The Lagrange method, being the most general and flexible, allows us to directly link the qualitative features of the dynamic behavior of a system to its fundamental symmetries. In particular, it allows us to consistently define and evaluate such important physical quantities as energy flow and energy velocity for complex systems. Finally, due to its generality and universality, the Lagrange formalism allows us to see the remarkable and profound similarity in apparently different physical systems, including discrete lattices of different dimensionality, as well as continuous media. One of the valuable applications of the nonreciprocal periodic electric 
network is thought to be a simulation - both mathematical and physical — of electromagnetic phenomena in periodic and almost periodic continuous media like photonic crystals. The only way to use our results for simulation purposes is to represent them in a universal "portable" form. Lagrange formalism provides us with such an opportunity. The well-known alternative to the Lagrange formalism is a traditional matrix approach to electric circuits analysis (see, for instance, [4]). Unfortunately, this traditional approach cannot be naturally carried over to an arbitrary nonreciprocal distributed system, for instance, magnetic photonic crystals. Of course, a simulation of distributed electromagnetic waves using lumped electric circuits should be carried out with caution and can be conducted only on the qualitative level.

2. Spectral nonreciprocity. Our investigation will focus on nonreciprocal periodic networks which can be viewed as periodic crystals with the corresponding spacetime symmetry, linear electric excitation spectrum, band structure, etc. So, in what follows we employ the terminology used in physics of crystalline solids; see, for instance, [1].

Linear excitation spectrum of an arbitrary periodic structure is a subject of Floquet-Bloch theory [1], [12], [11]. In the simplest case of a single band spectrum it is described by a single dispersion function $\Omega(\vec{k})$; here $\vec{k}$ is the Bloch number (the wave vector) of the eigenmode, $\Omega$ is the corresponding frequency. In our case the excitations are electric currents and voltages propagating through the mesh. In the one-dimensional case the wave vector $\vec{k}$ reduces to a scalar parameter $k$ which runs an interval

$$
-\frac{\pi}{a}<k \leq \frac{\pi}{a}
$$

where $a$ is the period of the structure. In the case of two- or three-dimensional structures, vector $\vec{k}$ varies within Brillouin zone of the corresponding dimensionality [1], [12].

In periodic systems with spectral reciprocity, any linear Bloch excitation $\Omega(\vec{k})$ has a reciprocal one with equal and opposite wave vector

$$
\Omega(\vec{k})=\Omega(-\vec{k}) .
$$

Our prime interest here will be with the property of spectral nonreciprocity

$$
\Omega(\vec{k}) \neq \Omega(-\vec{k})
$$

which is closely related (but not equivalent!) to the aforementioned electric nonreciprocity when the impedance matrix $Z$ is asymmetric. It turns out that the presence on nonreciprocal elements in a periodic electric circuit does not necessarily imply the spectral nonreciprocity (2.2). The inverse statement though is true: A periodic circuit with spectral nonreciprocity (2.2) must incorporate gyrators and thereby have an asymmetric $Z$-matrix.

Electric circuits with spectral nonreciprocity (2.2) display a number of remarkable features. For instance, an excitation with $\vec{k}=0$, often called a uniform excitation, does not necessarily correspond to a standing wave. Indeed, according to Figures 3.11 and 3.12 , the group velocity $\vec{u}(\vec{k})=\partial \Omega(\vec{k}) / \partial \vec{k}$ may not vanish at $\vec{k}=0$ :

$$
\vec{u}(0) \neq 0 \text {. }
$$

Therefore, in a mesh with spectral nonreciprocity, all or some of the excitations with $\vec{k}=0$ can transport the energy. The above effect gives a graphic manifestation of spectral nonreciprocity. 
3. Basic properties of spectrally nonreciprocal circuits. In this section we outline the basic properties of nonreciprocal periodic circuits and determine the dynamic origin of spectral nonreciprocity. Our consideration will be based on a particular example of a one-dimensional periodic electric circuit presented in Figure 3.3. This example features all the inherent characteristics of a general periodic system with spectral nonreciprocity. The justification of the basic properties of periodic nonreciprocal electric networks is considered in the following sections.

We begin with a brief analysis of the gyrator as a basic nonreciprocal network element.

3.1. The gyrator. The gyrator as a basic network element was introduced by Tellegen [15] (see also [4, section 5.1] and [8, Chapter 18]). Recall that in an electric circuit without gyrators the impedance matrix $Z$ is always symmetric [4, section 2.1].

$$
Z_{i j}=Z_{j i} .
$$

The property (3.1) of the impedance matrix $Z$ is a manifestation of the reciprocity principle [7, Chapters 3-8], [5, section 2.3]. The presence of gyrators may cause the impedance matrix to become asymmetric,

$$
Z_{i j} \neq Z_{j i}
$$

which can be interpreted as the property of nonreciprocity. Indeed, according to Tellegen [15], the ideal gyrator is defined as a lossless two-port network element with the following relations between the input and the output voltages $\left(v_{1}, v_{2}\right)$ and currents $\left(i_{1}, i_{2}\right)$ [4, section 5.1]:

$$
\left\{\begin{array}{l}
v_{1}=-\rho i_{2}, \\
v_{2}=\rho i_{1} .
\end{array}\right.
$$

The coefficient $\rho$ is called the gyration resistance. The corresponding impedance matrix of the gyrator is antisymmetric:

$$
Z=\left(\begin{array}{ll}
0 & \rho \\
-\rho & 0
\end{array}\right)=-Z^{T} .
$$

One of the physical realizations of the gyrator is presented in Figure 3.1. This device is based on the Hall effect. In conducting media, sufficiently strong magnetic field $\vec{H}$ causes the electrons to drift in the direction, perpendicular to the applied magnetic and electric fields. The corresponding density of macroscopic electric current is (see, for example, [16] and [17])

$$
\vec{J}=\frac{e n}{c} \vec{E} \times \vec{H},
$$

where $\vec{E}=\vec{E}(t)$ is an alternating electric field of the frequency $\omega, n$ is the density of conduction electrons or holes, $c$ is speed of light, and $e$ denotes the charge of each individual electric carrier (an electron or a hole). In Figure 3.1 the constant magnetic field $\vec{H}$ points in the $z$-direction; the alternating electric field $\vec{E}(t)$ lies within the $x y$-plane. In this case the regular electric conductivity when $\vec{J}$ is parallel to $\vec{E}$ is suppressed, and the remaining lossless Hall conductivity (3.5) causes the impedance matrix of the device to take the form (3.4). The actual design of the device can be 


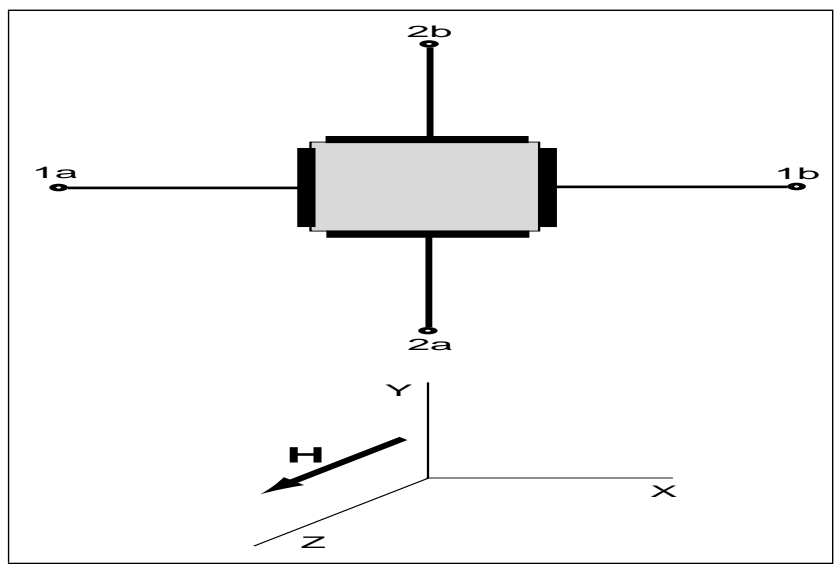

FIG. 3.1. Gyrator based on the Hall effect. The macroscopic electric current $\mathbf{J}$ in the conducting medium is perpendicular to the strong steady magnetic field $\mathbf{H} \| \mathbf{Z}$ and to the alternating electric field $\mathbf{E}(t)$. This representation adequately reflects the actual symmetry of the physical device.

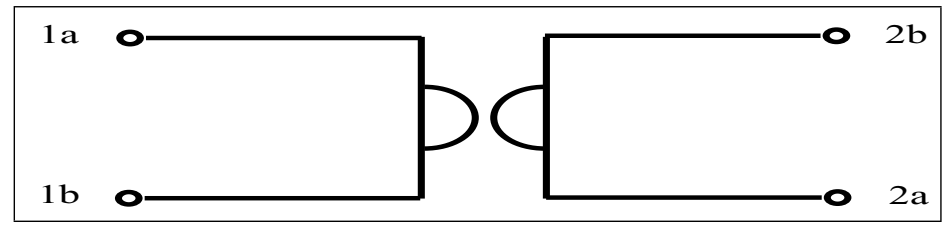

FIG. 3.2. Conventional graphic representation of the gyrator as a two-port network element. This representation does not adequately reflect the gyrator's actual symmetry.

different, but in any case the alternating electric field $\vec{E}$ must be strictly perpendicular to $\vec{H}$ for the diagonal components $Z_{11}$ and $Z_{22}$ in (3.4) to be zeros.

Practically, the ideal situation (3.4) can be easily realized in some pure singlecrystalline metals, like copper, at low temperatures, when the mean-free path of electric carriers can reach as much as one centimeter or more (see [16], [17], and references therein). The required magnitude of the $d c$ magnetic field $\vec{H}$ does not exceed a fraction of a Tesla. In devices based on the Hall effect in semiconductors the ideal situation (3.4) cannot even be approached because the diagonal components $Z_{11}$ and $Z_{22}$ cannot be effectively suppressed. At microwave range, the most popular gyrator design is based on the effect of Faraday rotation in ferrites [8].

Figure 3.2 represents the conventional picture of the gyrator as a two-port network element [4].

3.2. Lagrangian of a gyrotropic system. Let us start with general setting. For a dynamical system described by coordinates $Q=\left\{q_{i}\right\}$ and time $t$, the time reversal symmetry can be formulated as follows. For any trajectory $Q(t)$ of the system, $\tilde{Q}(t)=Q(-t)$ is also its trajectory. In terms of the Lagrangian function $\mathcal{L}=\mathcal{L}(Q, \dot{Q})$ it means the invariance of $\mathcal{L}$ under the transformation $\dot{Q} \rightarrow-\dot{Q}$ :

$$
\mathcal{L}(Q, \dot{Q})=\mathcal{L}(Q,-\dot{Q})
$$




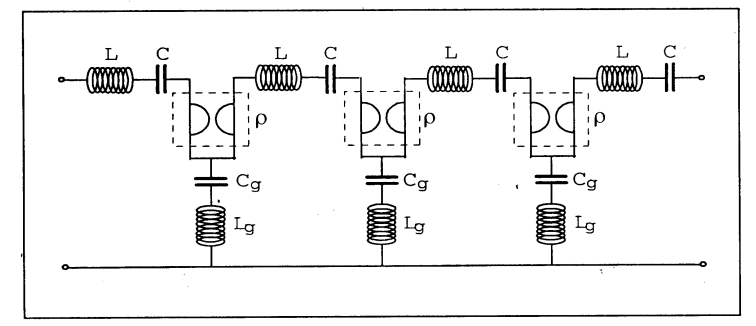

FIG. 3.3. A typical example of a periodic circuit with a single-band nonreciprocal spectrum.

Respectively, for a system with broken time reversal symmetry we have

$$
\mathcal{L}(Q, \dot{Q}) \neq \mathcal{L}(Q,-\dot{Q})
$$

If $\mathcal{L}$ is a general bilinear form of all its variables $\left\{q_{i}\right\}$ it takes the form

$$
\begin{aligned}
\mathcal{L} & =T+G-V, \\
T & =\sum_{i, j} T_{i, j} \dot{q}_{i} \dot{q}_{j}, V=\sum_{n, m} V_{i, j} q_{i} q_{j}, G=\sum_{i, j} G_{i, j} \dot{q}_{i} q_{j} .
\end{aligned}
$$

Assuming that each of the coordinates $\left\{q_{i}\right\}$ is either a time-even or time-odd physical quantity, one can state that the violation of the time reversal symmetry occurs if the gyroscopic term $G$ does not vanish [13]. In our consideration, each coordinate is the electric charge

$$
q_{i}=\int j_{i}(t) d t
$$

where $j_{i}$ is the corresponding electric current. Therefore, all the coordinates $\left\{q_{i}\right\}$ are time-even physical quantities.

In the case of energy conservative systems the natural causes for the broken time reversal symmetry are the forces perpendicular to the velocities, i.e., the forces that perform no work. The classical and the simplest examples of the kind are the Lorentz and the Coriolis forces.

3.3. Example of an electric circuit with spectral nonreciprocity. Let us consider a periodic electric circuit depicted in Figure 3.3. A periodic electric mesh can be viewed as a periodic array of elementary circuits labeled by integer valued index $n$ running one-dimensional lattice

$$
\check{Z}^{1}=\{\ldots,-2,-1,0,1,2, \ldots\} .
$$

As we will see later, the particular example in Figure 3.3 represents the simplest circuit that features virtually all qualitative characteristics of periodic electric circuits with spectral nonreciprocity. For a better understanding of general properties of our systems we study the Lagrangian and the Hamiltonian of the system. This gives us flexibility in the choice of variables and allows us to find the representation for such 
fundamental physical quantities as the site energy, the site energy flow, and more. The Lagrangian of the circuit in Figure 3.3 is

$$
\begin{aligned}
\mathcal{L}= & \sum_{n} \frac{L \dot{Q}_{n}^{2}}{2}-\frac{Q_{n}^{2}}{2 C}+\frac{\rho\left(Q_{n-1} \dot{Q}_{n}-Q_{n} \dot{Q}_{n-1}\right)}{2} \\
& +\frac{L_{g}\left(\dot{Q}_{n}-\dot{Q}_{n-1}\right)^{2}}{2}-\frac{\left(Q_{n}-Q_{n-1}\right)^{2}}{2 C_{g}},
\end{aligned}
$$

where $Q_{n}$ is the "charge," i.e., the time integral of the electric current, in the $n$th site of the circuit. $L, L_{g}, C$, and $C_{g}$ denote the corresponding inductances and capacitances. One can make sure that the "nonreciprocal" term in the Lagrangian (3.10) is consistent with the impedance equations (3.3) for the gyrator.

3.4. Dispersion relations. As follows from the computations in section 6 , the dispersion relation for the circuit in Figure 3.3 reduces to a quadratic equation

$$
\omega^{2}+2 \frac{\rho \sin (k)}{\hat{\alpha}(k)} \omega-\frac{\hat{\beta}(k)}{\hat{\alpha}(k)}=0,
$$

where

$$
\hat{\alpha}(k)=L+2 L_{g}(1-\cos (k)), \hat{\beta}(k)=\frac{1}{C}+\frac{2}{C_{g}}(1-\cos (k)) .
$$

The explicit dispersion relation is the positive solution $\Omega(k)$ of the characteristic equation (3.11):

$$
\Omega(k)=\frac{\sqrt{[\rho \sin (k)]^{2}+\hat{\alpha}(k) \hat{\beta}(k)}-\rho \sin (k)}{\hat{\alpha}(k)} .
$$

The spectrum of resonant frequencies for the circuit is then just an interval

$$
\left[\Omega_{-}, \Omega_{+}\right], \Omega_{-}=\min _{-\pi \leq k \leq \pi} \Omega(k), \Omega_{+}=\max _{-\pi \leq k \leq \pi} \Omega(k) .
$$

The explicit expressions for the band edges $\Omega_{ \pm}$are

$$
\Omega_{ \pm}^{2}=\frac{\left(a c+L b+4 \rho^{2}\right) \pm \sqrt{\left(a c+L b+4 \rho^{2}\right)^{2}-4 L a c b}}{2 L a},
$$

where

$$
a=\left(L+4 L_{g}\right), b=\frac{1}{C}+\frac{4}{C_{g}}, c=\frac{1}{C} .
$$

Typical dispersion relations are displayed in Figures 3.4 and 3.5.

The case presented in Figure 3.5 exhibits qualitatively different behavior in the vicinity of $k=0$.

It is important that the presence of gyrators in a periodic electric network does not guarantee the property of spectral nonreciprocity. Indeed, in Figures 3.6 and 3.7 we presented two different periodic meshes built from the same nonreciprocal elementary fragments (primitive cells) presented in Figure 3.8. 


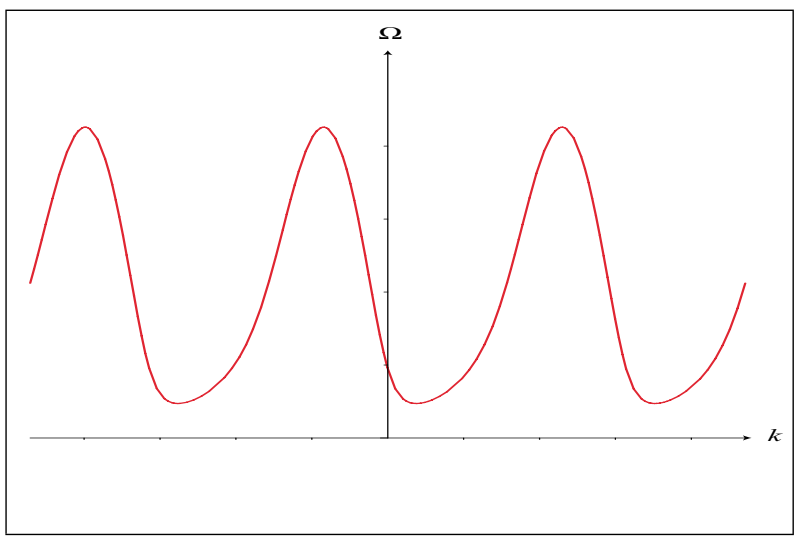

FIG. 3.4. A single-band nonreciprocal spectrum corresponding to a finite $C$. In this case, $\Omega(k) \neq 0$ at $k=0$.

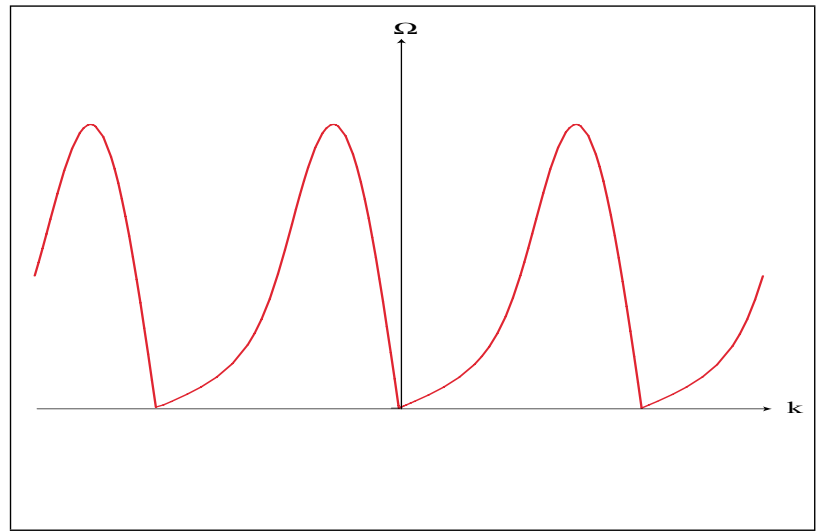

FIG. 3.5. A single-band nonreciprocal spectrum corresponding to $C=\infty$. In this case, $\Omega(k)=0$ at $k=0$.

The elementary fragment in Figure 3.8 is a simple nonreciprocal resonator composed of a gyrator with either port shunted by the same inductance $L$. Taken separately, it would have the resonance frequency

$$
\Omega_{0}=\rho L^{-1} .
$$

As long as the mutual inductance $M$ between the neighboring coils is taken into account, the spectrum of free electric oscillations can be represented in the Bloch form $\Omega(\vec{k})$. In particular, the dispersion relations for the circuits in Figures 3.6 and 3.7 , respectively, are

$$
\begin{aligned}
& \Omega(k)=\rho\left(\sqrt{L^{2}-M^{2} \cos ^{2} k}-M \sin k\right)^{-1}, \\
& \Omega(k)=\rho\left(\sqrt{L^{2}-4 M L \cos k}\right)^{-1} .
\end{aligned}
$$

From these representation of the dispersion relations we can readily see that the circuit in Figure 3.6 displays the property (2.2) of spectral nonreciprocity whereas the one in Figure 3.7 has a reciprocal spectrum satisfying (2.1). At the same time, both circuits have asymmetric impedance matrixes $Z_{i j}$. 


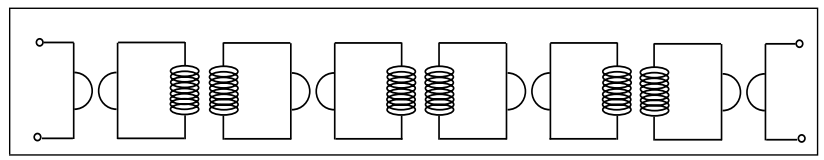

FIG. 3.6. First example of a one-dimensional periodic network with the primitive cell shown in Figure 3.8. The symmetry of this mesh is compatible with spectral nonreciprocity.

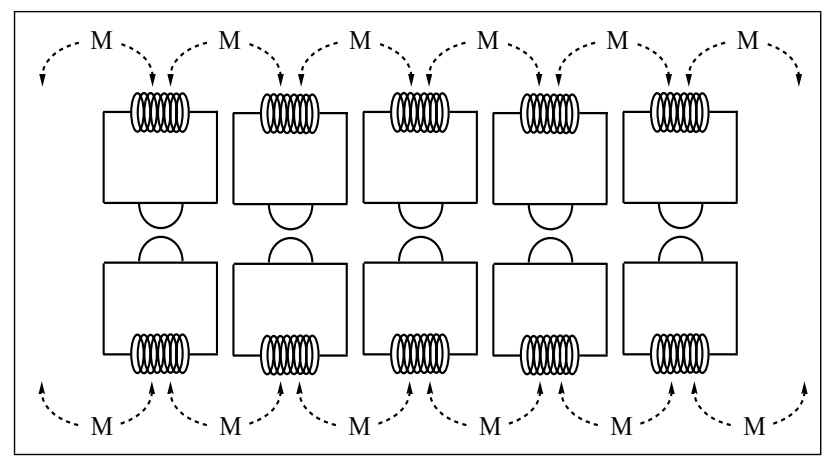

FIG. 3.7. Second example of a one-dimensional periodic network with the same primitive cell shown in Figure 3.8. The symmetry of this mesh is incompatible with spectral nonreciprocity.

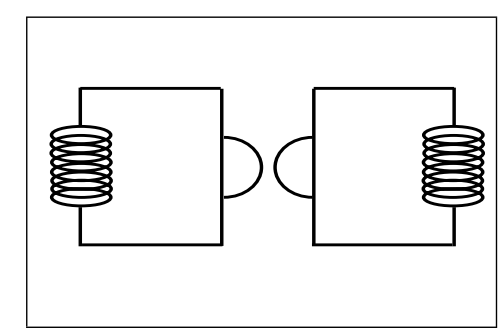

FIG. 3.8. A nonreciprocal resonant circuit which is the prime cell of the infinite periodic networks presented in Figures 3.6 and 3.7.

3.5. Eigenmodes. Let us turn back to the circuit in Figure 3.3. The evolution equation in this case is

$$
\begin{aligned}
L \ddot{Q}_{n} & +L_{g}\left(2 \ddot{Q}_{n}-\ddot{Q}_{n+1}-\ddot{Q}_{n-1}\right)+\rho\left(\dot{Q}_{n-1}-\dot{Q}_{n+1}\right)+\frac{Q_{n}}{C} \\
& +\frac{2 Q_{n}-Q_{n+1}-Q_{n-1}}{C_{g}}=0, n \text { in } \check{Z}^{1} .
\end{aligned}
$$

For the time harmonic $Q_{n}(t)=e^{i \omega t} Q_{n}$, (3.19) takes the form of the second-order difference equation

$$
\begin{aligned}
& -\omega^{2}\left[L Q_{n}+L_{g}\left(2 Q_{n}-Q_{n+1}-Q_{n-1}\right)\right]+i \omega \frac{\rho\left(Q_{n-1}-Q_{n+1}\right)}{2}+\frac{Q_{n}}{C} \\
& +\frac{\left(2 Q_{n}-Q_{n+1}-Q_{n-1}\right)}{C_{g}}=0, \quad n \text { in } \check{Z}^{1} .
\end{aligned}
$$


After simplification it becomes

$$
b_{0}(\omega) Q_{n}+b_{1}(\omega) Q_{n+1}+b_{1}(-\omega) Q_{n-1}=0
$$

where

$$
\begin{aligned}
& b_{0}(\omega)=\frac{1}{C}+\frac{2}{C_{g}}-\left(L+2 L_{g}\right) \omega^{2}, \\
& b_{1}(\omega)=L_{g} \omega^{2}-\frac{1}{C_{g}}-i \rho \omega .
\end{aligned}
$$

Observe that for real $\omega$

$$
b_{1}(-\omega)=\overline{b_{1}(\omega)} \text {. }
$$

Note also that (3.21) is an equation for the eigenmodes associated with frequency $\omega$. Since (3.21) is a second-order homogeneous difference equation with constant coefficients, we may seek its solutions in the exponential form

$$
Q_{n}=z^{-n}, z=e^{i k},
$$

where $z$ is the propagation factor and $k$ is the wave number which may take complex values. Plugging the sequence (3.25) in (3.21) we arrive at the following equation for the propagation factor $z$ :

$$
b_{0}(\omega)+b_{1}(\omega) z^{-1}+b_{1}(-\omega) z=0 .
$$

Recall that a sequence $Q_{n}$ which solves (3.21) is an eigenmode of the frequency $\omega$. Hence, for every frequency $\omega$ we have exactly two eigenmodes $\Psi_{n}^{ \pm}(\omega)$

$$
\begin{aligned}
\Psi_{n}^{ \pm}(\omega) & =\left[Z_{ \pm}(\omega)\right]^{-n} \\
Z_{ \pm}(\omega) & =\exp \left[i K_{ \pm}(\omega)\right]
\end{aligned}
$$

where $Z_{ \pm}(\omega)$ are the two solutions of the quadratic equation (3.26). Observe that for the frequencies in the spectrum $\left[\Omega_{-}, \Omega_{+}\right]$the wave numbers $K_{ \pm}(\omega)$ are real.

Since (3.26) is just another form of the dispersion equation (3.11) for $z=e^{i k}$, it will be referred to as the dispersion relation as well. In addition to that we have

$$
\Omega\left(K_{ \pm}(\omega)\right)=\omega
$$

where $\Omega(k)$ is defined by (3.11).

For the time-dependent eigenmodes we use one of the following representations:

$$
\Psi_{n}(k)=\exp [i(\Omega(k) t-k n)],-\pi<k \leq \pi,
$$

Or

$$
\Psi_{n}^{ \pm}(\omega)=\exp \left[i\left(\omega t-K_{ \pm}(\omega) n\right)\right], \Omega_{-} \leq \omega \leq \Omega_{+} .
$$

Having these representations we define the phase and group velocities by standard formulas. Namely, the phase velocity is $v=\omega / k$, while the group velocity is $u=$ $d \omega / d k$. More exactly,

$$
v(k)=\frac{\Omega(k)}{k}, u(k)=\frac{d \Omega(k)}{d k},-\pi<k \leq \pi .
$$


Consequently, the frequency dependence of the phase and the group velocities are expressed by the formulas

$$
\begin{aligned}
& v_{ \pm}(\omega)=\frac{\omega}{K_{ \pm}(\omega)} \\
& u_{ \pm}(\omega)=\left[\frac{d K_{ \pm}(\omega)}{d \omega}\right]^{-1} .
\end{aligned}
$$

3.6. Phase and group velocities. An evident manifestation of the spectral nonreciprocity is that for $\rho \neq 0$ we have

$$
\begin{gathered}
v_{+}(\omega) \neq-v_{-}(\omega) ; \\
u_{+}(\omega) \neq-u_{-}(\omega) ; \\
K_{+}(\omega) \neq-K_{-}(\omega) .
\end{gathered}
$$

This can be readily seen from the explicit representation (3.13) for $\Omega(k)$. Moreover, the wave numbers $K_{+}(\omega)$ and $K_{-}(\omega)$ as well as the corresponding phase velocities $v_{+}(\omega)$ and $v_{-}(\omega)$ may even have the same sign for some frequencies in the spectrum. The group velocities $u_{+}(\omega)$ and $u_{-}(\omega)$ though must always have different signs unless they are zeros (see section 6). We use this important property of the group velocity to set from now on the indices \pm as follows:

$$
u_{+}(\omega) \geq 0, u_{-}(\omega) \leq 0
$$

Then $K_{+}(\omega)$ and $K_{-}(\omega)$ are set by the relations (3.34). The indices \pm for phase velocities are consequently defined by (3.33). For the wave numbers $K_{ \pm}(\omega)$ the following representation holds:

$$
\begin{aligned}
K_{ \pm}(\omega) & =\mp K_{0}(\omega)+\varkappa(\omega), \\
K_{0}(\omega) & =\arccos \left(-\frac{b_{0}(\omega)}{2\left|b_{1}(\omega)\right|}\right), \\
\varkappa(\omega) & =\arctan \frac{\rho \omega}{L_{g} \omega^{2}-\left(1 / C_{g}\right)},
\end{aligned}
$$

where we choose

$$
0 \leq \arccos (t) \leq \pi,-\pi / 2 \leq \arctan (t) \leq \pi / 2 .
$$

The wave vectors $K_{ \pm}(\omega)$ are plotted in Figures 3.9 and 3.10.

Based on the dispersion relation (3.13) and (3.12) one can derive the following formulas for the group velocity $u$ and the phase velocity $v$ :

$$
u(k)=\frac{d \Omega(k)}{d k}=\frac{u_{n}}{u_{d}},
$$

where

$$
\begin{aligned}
& u_{n}=\left[\left(L / C_{g}\right)-\left(L_{g} / C\right)\right] \sin (k)+[\hat{\alpha}(k)-L(1+\cos (k))] \rho \Omega(k), \\
& u_{d}=\hat{\alpha}(k)[\hat{\alpha}(k) \Omega(k)+\rho \sin (k)],
\end{aligned}
$$

and

$$
v(k)=\frac{\Omega(k)}{k} .
$$

The frequency-dependent velocities $u_{ \pm}(\omega)$ and $v_{ \pm}(\omega)$ can be found by the formulas (3.33) and (3.34) where $K_{ \pm}(\omega)$ is given by (3.39), (3.40), (3.41). The typical behavior of the group velocity is shown in Figures 3.11 and 3.12 . 


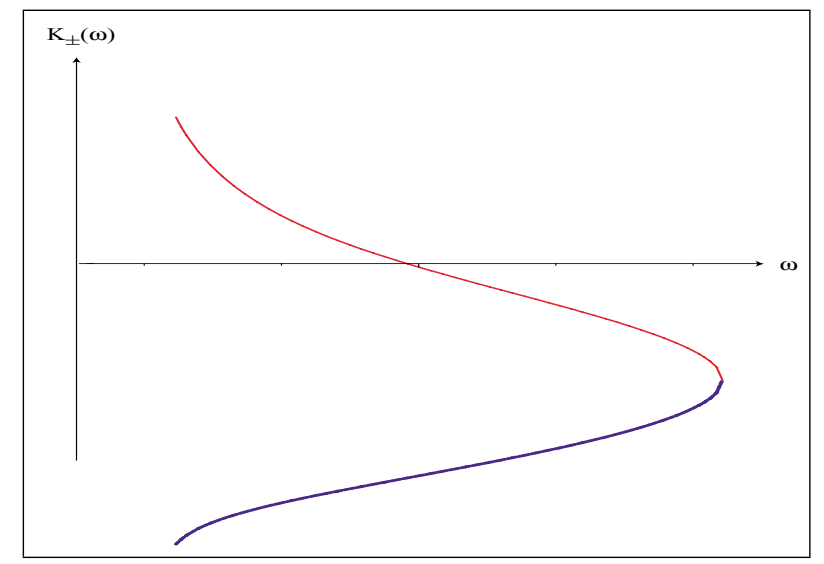

FIG. 3.9. Typical dependence $K \pm(\omega)$ for the case of finite $C$.

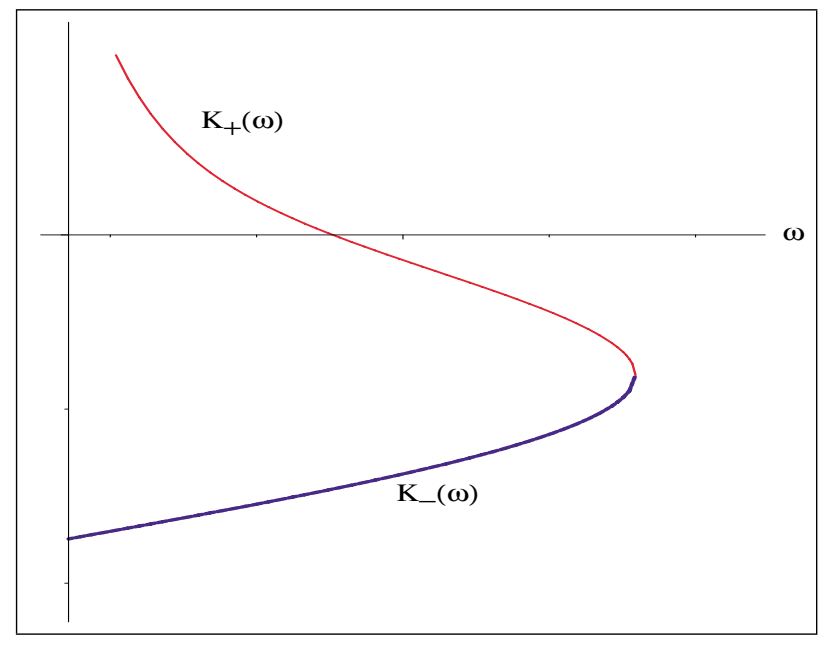

FIG. 3.10. $K_{ \pm}(\omega)$ for the case $C=\infty$.

3.7. Energy flow and energy velocity. Consider now the case when $Q_{n}=$ $\Psi_{n}(k)$ is the eigenmode of the form (3.30). Let $E(k)$ and $S(k)$ be the time average of the instant energy and energy flow at any site $n$, respectively (see detailed analysis of these quantities in sections 6.4 and 6.5). In the case of the circuit in Figure 3.3 the explicit formulas can be derived for $E(k)$ and $S(k)$. For the energy we have

$$
E(k)=\frac{1}{4}\left[\hat{\alpha}(k) \Omega(k)^{2}+\hat{\beta}(k)\right]
$$

or, using the dispersion relation (3.13), we recast (3.45) as

$$
E(k)=\frac{5}{16 C}+\frac{5(1-\cos k)}{8 C_{g}}-\frac{\rho}{4} \Omega(k) \sin k .
$$

The energy flow then is

$$
S(k)=\frac{\Omega(k)}{2}\left[\frac{\sin k}{C_{g}}-\Omega(k) \rho \cos k-\Omega(k)^{2} L_{g} \sin k\right] .
$$




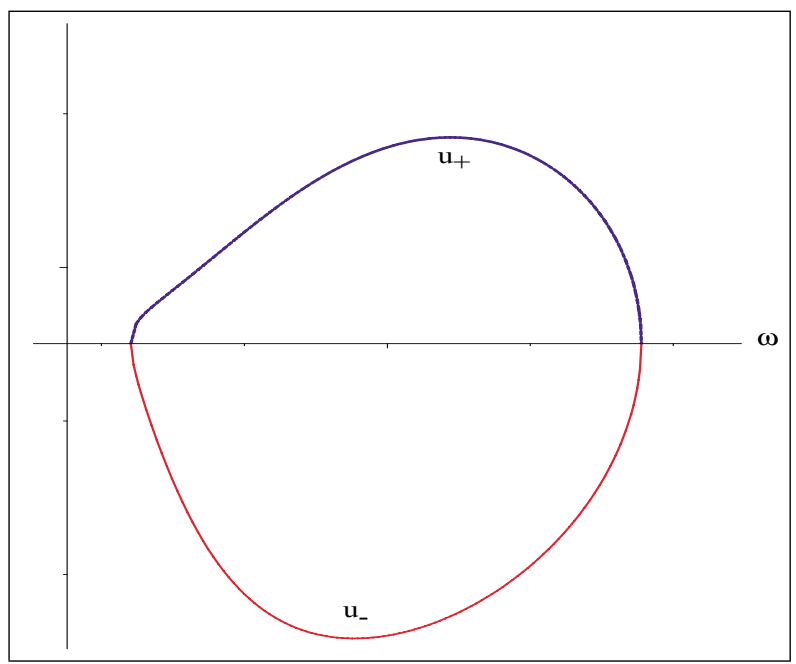

FIG. 3.11. Frequency dependence of the group velocity for the case of finite $C$.

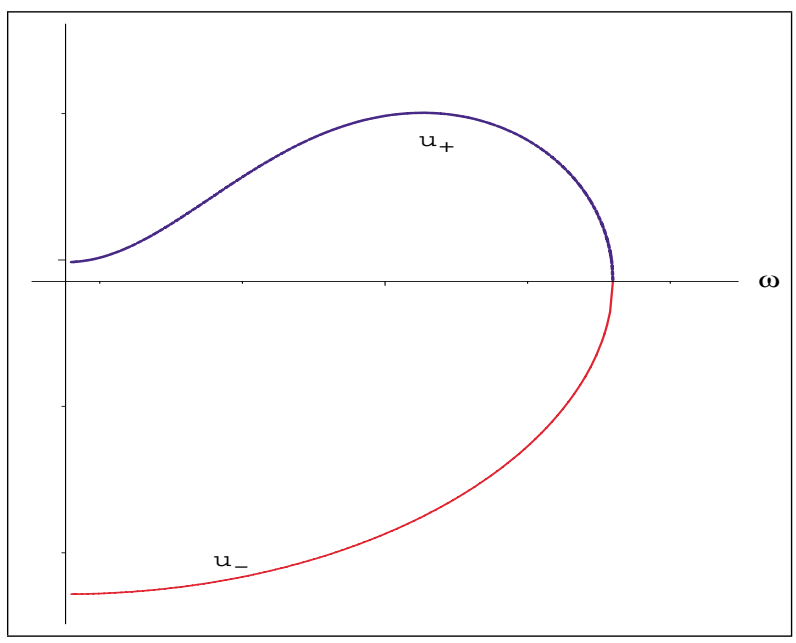

FIG. 3.12. Frequency dependence of the group velocity for the case $C=\infty$.

Finally, the energy velocity is

$$
w(k)=\frac{S(k)}{E(k)}=\frac{8 \Omega(k)\left[C_{g}^{-1} \sin k-\Omega(k) \rho \cos k-\Omega(k)^{2} L_{g} \sin k\right]}{5 C^{-1}+10 C_{g}^{-1}(1-\cos k)-4 \Omega(k) \rho \sin k} .
$$

From this we get the following formula for the frequency-dependent energy velocity:

$$
w_{ \pm}(\omega)=w\left(K_{ \pm}(\omega)\right)
$$

where $K_{ \pm}(\omega)$ is given by (3.39), (3.40), (3.41). A typical behavior of the energy velocity $w_{ \pm}(\omega)$ shows close resemblance to the group velocity $u_{ \pm}(\omega)$ that is a common characteristic of all spatially periodic systems. 
3.8. Green's function. The Green's function $G_{n}$ is defined as a special solution to the following inhomogeneous modification of (3.20):

$$
b_{0}(\omega) G_{n}+b_{1}(\omega) G_{n+1}+b_{1}(-\omega) G_{n-1}=\delta_{0, n},
$$

where $\delta_{0, n}$ is the Kronecker symbol. One can verify that the function $G_{n}$ defined below solves (3.50):

$$
G_{n}=\frac{1}{g_{0}}\left\{\begin{array}{lll}
{\left[Z_{+}(\omega)\right]^{-n}} & \text { if } \quad n>0, \\
{\left[Z_{-}(\omega)\right]^{-n}} & \text { if } \quad n \leq 0,
\end{array}\right.
$$

where

$$
\begin{aligned}
g_{0}= & -\omega^{2}\left[L+L_{g}\left(2-Z_{+}^{-1}-Z_{-}\right)\right]+i \omega \rho\left(Z_{-}-Z_{+}^{-1}\right) \\
& +\frac{1}{C}+\frac{2-Z_{+}^{-1}-Z_{-}}{C_{g}} ; Z_{ \pm}=Z_{ \pm}(\omega)=e^{i K_{ \pm}(\omega)} .
\end{aligned}
$$

Note that the solution to (3.50) is defined up to a general solution to the homogeneous equation (3.20). We remind the reader that in the case of spectrally reciprocal media the Green's function is selected from a set of solutions by an additional requirement. This requirement is that the Green's function must satisfy the causality condition which in this case is equivalent to the so-called outgoing wave condition as $|n| \rightarrow \infty$ [5]. In our case the condition is reduced to the requirement that the Green's function $G_{n}$ must behave as a wave with a positive group velocity for $n>0$ and a negative group velocity for $n<0$. This condition singles out the unique solution to the inhomogeneous equation (3.50) of the form (3.51), (3.52).

Recall that the Green's function describes the envelope of the waves generated by alternating external force with frequency $\omega$. The formula (3.51) for the Green's function along with (3.36) imply that energy propagation will be asymmetric according to the asymmetry in the two group velocities $u_{ \pm}(\omega)$. The degree of spectral asymmetry can be very significant. According to (3.5) in the case of the gyrator based on the Hall effect, the gyration resistance $\rho$ is proportional to $\frac{e}{c} n H$, and it can vary over wide limits.

4. Finite cyclic circuits. Generally, a finite cyclic circuit of $N$ blocks can be viewed as a periodic infinite circuit similar to that shown in Figure 3.3 with an additional condition

$$
Q_{N}=Q_{0}
$$

imposed on it. It has $N$ degrees of freedom, namely, the charges

$$
Q_{0}, \ldots, Q_{N-1},
$$

and is governed by (3.19) subject to the cyclic condition (4.1). In this case the discrete Fourier transform employs the wave numbers of the form

$$
k_{m}=\frac{2 \pi m}{N}, m=0, \ldots, N-1 .
$$

For every $k_{m}$ we have the eigenfrequency $\Omega\left(k_{m}\right)$ and the corresponding eigenmode

$$
\Psi_{n}\left(k_{m}\right)=\exp \left\{i\left(\Omega\left(k_{m}\right) t-k_{m} n\right)\right\}, n=0, \ldots, N-1 .
$$


The energy velocity for this eigenmode will be simply $w\left(k_{m}\right)$ where $w(k)$ is given by (3.48). As to the group velocity, in view of (4.3) the wave number takes on discrete values and, hence, formally we cannot use the standard definition of the group velocity as $\Omega^{\prime}(k)$. Instead we can use the expressions

$$
v_{N}\left(k_{m}\right)=\frac{\Omega\left(k_{m+1}\right)-\Omega\left(k_{m}\right)}{k_{m+1}-k_{m}} .
$$

Note that typically all the frequencies $\Omega\left(k_{m}\right), m=0, \ldots, N-1$ are different and, hence, every eigenfrequency is associated with a particular direction of propagation, either clockwise and counterclockwise, depending on the sign of, say, the corresponding energy velocity $w\left(k_{m}\right)$.

5. Lagrangian and Hamiltonian functions. In this section we consider the general and special Lagrangian and Hamiltonian functions associated with our nonreciprocal (gyroscopic) systems.

Let us introduce first an elementary system associated with an oscillator having $\nu$ degrees of freedom described by a real valued $\nu$-dimensional vector $q$. We also introduce a set of indices, lattice, $\breve{S}$. $\breve{S}$ can be, for instance, one-dimensional lattice $\check{Z}^{1}$ or multidimensional lattice $\check{Z}^{d}, d=2,3$, or the continuous space $\check{R}^{d}, d=1,2,3$. Then we attach to every site $n$ in $\check{S}$ an oscillator, i.e., $\nu$-dimensional vector $q_{n}$. The system of oscillators is

$$
Q=\left\{q_{n}, n \text { in } \check{S}\right\}, q_{n} \text { in } \check{R}^{\nu} .
$$

Assuming then the Lagrangian function $\mathcal{L}$ of the system to be a bilinear (quadratic) form we represent it as

$$
\begin{aligned}
& \mathcal{L}=\frac{1}{2}\left[\begin{array}{l}
\dot{Q} \\
Q
\end{array}\right]^{T}\left[\begin{array}{ll}
\alpha & \zeta \\
\zeta^{T} & -\beta
\end{array}\right]\left[\begin{array}{l}
\dot{Q} \\
Q
\end{array}\right], \\
& \alpha=\alpha^{T}, \bar{\alpha}=\alpha, \beta=\beta^{T}, \bar{\beta}=\beta, \bar{\zeta}=\zeta,
\end{aligned}
$$

where the subscript " $T$ " denotes the matrix transposition, the matrices $\alpha$ and $\beta$ are positive Hermitian matrices, and $\zeta$ is an arbitrary matrix. All the matrices $\alpha, \beta$, and $\zeta$ are assumed to have real valued entries. Observe that the matrix entries $\alpha_{n, m}$, $\beta_{n, m}$, and $\zeta_{n, m}$ are $\nu \times \nu$-matrices such that

$$
\begin{aligned}
& \bar{\alpha}_{n, m}=\alpha_{n, m}, \bar{\beta}_{n, m}=\beta_{n, m}, \bar{\zeta}_{n, m}=\zeta_{n, m}, \\
& \alpha_{n, m}^{T}=\alpha_{m, n}, \beta_{n, m}=\beta_{m, n} .
\end{aligned}
$$

In other words,

$$
\begin{aligned}
\mathcal{L} & =\mathcal{L}(Q, \dot{Q})=\frac{1}{2}\left[\dot{Q}^{T} \alpha \dot{Q}+\dot{Q}^{T} \zeta Q+Q^{T} \zeta^{T} \dot{Q}-Q^{T} \beta Q\right] \\
& =\frac{1}{2} \dot{Q}^{T} \alpha \dot{Q}+\dot{Q}^{T} \zeta Q-\frac{1}{2} Q^{T} \beta Q
\end{aligned}
$$

The Euler-Lagrange evolution equation for the system is

$$
\frac{d}{d t}\left(\frac{\partial \mathcal{L}}{\partial \dot{Q}}\right)-\frac{\partial \mathcal{L}}{\partial Q}=0
$$


For $\mathcal{L}$ defined by $(5.6)$ it takes the form

$$
\alpha \ddot{Q}+\left(\zeta-\zeta^{T}\right) \dot{Q}+\beta Q=0 .
$$

It follows from (5.8) that the necessary and sufficient condition of the spectral nonreciprocity is

$$
\zeta \neq \zeta^{T}
$$

Indeed, if $\zeta=\zeta^{T}$, a gyroscopic system will not exhibit spectral nonreciprocity. The reason is that in the case $\zeta=\zeta^{T}$ the term with $\dot{Q}$ vanishes from (5.8) and all the frequencies related to (5.8) will be the same as for the case of zero $\zeta$.

To find the Hamiltonian function of the system we set

$$
H(P, Q)=\frac{1}{2} X^{T} A_{H} X, X=\left[\begin{array}{l}
P \\
Q
\end{array}\right] .
$$

The Hamiltonian equations for the time evolution then take the form

$$
\begin{aligned}
\dot{X} & =D_{H} X, D_{H}=J A_{H}, \\
J & =\left[\begin{array}{ll}
0 & -\check{I} \\
\check{I} & 0
\end{array}\right],
\end{aligned}
$$

where $\check{I}$ is the identity operator.

The relation between $\mathcal{L}$ and $H$ is

$$
H(P, Q)=P^{T} \dot{Q}-\mathcal{L}(Q, \dot{Q}),
$$

where

$$
P=\left(\frac{\partial \mathcal{L}}{\partial \dot{Q}}\right)^{T}=\alpha \dot{Q}+\zeta Q
$$

and, hence,

$$
\dot{Q}=\alpha^{-1}(P-\zeta Q) .
$$

Observe that in the nonreciprocal case $\zeta \neq 0$ the relation between the momentum $P$ and the "velocity" $\dot{Q}$ depend on $Q$. From (5.13) and (5.14) we obtain

$$
\begin{aligned}
& H(P, Q)=(\alpha \dot{Q}+\zeta Q)^{T} \dot{Q}-\left(\frac{1}{2} \dot{Q}^{T} \alpha \dot{Q}+\dot{Q}^{T} \zeta Q-\frac{1}{2} Q^{T} \beta Q\right) \\
= & \frac{1}{2}\left(\dot{Q}^{T} \alpha \dot{Q}+Q^{T} \beta Q\right) \\
= & \frac{1}{2}\left[(P-\zeta Q)^{T} \alpha^{-1}(P-\zeta Q)+Q^{T} \beta Q\right] \\
= & \frac{1}{2} P^{T} \alpha^{-1} P-Q^{T} \zeta^{T} \alpha^{-1} P-P^{T} \alpha^{-1} \zeta Q+Q^{T}\left(\zeta^{T} \alpha^{-1} \zeta+\beta\right) Q
\end{aligned}
$$

which, in particular, imply $H(P, Q) \geq 0$. Based on (5.16) we find that the operator $A_{H}$ in (5.10) is

$$
A_{H}=\left[\begin{array}{ll}
\alpha^{-1} & -\alpha^{-1} \zeta \\
-\zeta^{T} \alpha^{-1} & \zeta^{T} \alpha^{-1} \zeta+\beta
\end{array}\right] .
$$


This, in turn, implies the following representation for operator $D_{H}$ in the Hamilton equation (5.11):

$$
\begin{aligned}
D_{H} & =J A_{H}=\left[\begin{array}{ll}
0 & -\check{I} \\
\check{I} & 0
\end{array}\right]\left[\begin{array}{ll}
\alpha^{-1} & -\alpha^{-1} \zeta \\
-\zeta^{T} \alpha^{-1} & \zeta^{T} \alpha^{-1} \zeta+\beta
\end{array}\right] \\
& =\left[\begin{array}{ll}
\zeta^{T} \alpha^{-1} & -\zeta^{T} \alpha^{-1} \zeta-\beta \\
\alpha^{-1} & -\alpha^{-1} \zeta
\end{array}\right] .
\end{aligned}
$$

Summarizing, we find from (5.10) and (5.18) that the system evolution is governed by the equation

$$
\begin{aligned}
\dot{X} & =D_{H} X \\
D_{H} & =\left[\begin{array}{ll}
\zeta^{T} \alpha^{-1} & -\zeta^{T} \alpha^{-1} \zeta-\beta \\
\alpha^{-1} & -\alpha^{-1} \zeta
\end{array}\right] .
\end{aligned}
$$

\section{Dispersive properties of general nonreciprocal circuits.}

6.1. Dispersion relations. Let us investigate the spectral properties of (5.19) and matrix operator (5.20) for the circuit in Figure 3.3. Note that the circuit is a homogeneous $\check{Z}^{1}$ lattice medium. Hence, the plane lattice waves of the form $\exp (i k n)$ are the eigenmodes of the system. This, in particular, implies that the Fourier transform diagonalizes the matrix operator $D_{H}$. This fact, in turn, enables us to find the spectrum, eigenmodes, dispersion relations, and other important quantities analytically. We remind the reader that in the case of the circuits the degrees of freedom are described by the sequence of charges $Q=\left\{Q_{n}, n\right.$ in $\left.\check{Z}^{1}\right\}$.

Before applying the Fourier transform for (5.19) and (5.20) with respect to index $n$ in $\check{Z}^{1}$ we define the shift operator $U$ by

$$
(U Q)_{n}=Q_{n-1}
$$

In other words, the entries of the matrix $U$ are

$$
U_{n, m}=\delta_{n-1, m}, n, m \text { in } \check{Z}^{1},
$$

where $\delta_{n, m}$ is the Kronecker's symbol. Observe also that

$$
\begin{aligned}
\left(U^{s} Q\right)_{n} & =Q_{n-s}, \quad s=0, \pm 1, \pm 2, \ldots, \\
U^{T} & =U^{-1}
\end{aligned}
$$

If we introduce now the Fourier transform

$$
\begin{aligned}
\hat{Q}(k) & =\sum_{n=-\infty}^{\infty} Q_{n} e^{i k n}, \\
Q_{n} & =\frac{1}{2 \pi} \int_{-\pi}^{\pi} \hat{Q}(k) e^{-i k n} d k,
\end{aligned}
$$

then the Fourier transform $\hat{U}$ of the operator $U$ is

$$
[\hat{U} \hat{Q}](k)=e^{i k} \hat{Q}(k) .
$$

In other words, $\hat{U}$ is the operator of the multiplication by the function

$$
\hat{U}(k)=e^{i k}
$$


and, hence,

$$
\hat{U}^{s}(k)=e^{i s k} .
$$

Applying now to the circuit Lagrangian function (3.10) the general representation (5.6) we find the following expressions for $\alpha, \beta$, and $\zeta$ :

$$
\begin{aligned}
& \alpha=L \breve{I}+L_{g}\left(\breve{I}-U^{-1}\right)(\breve{I}-U)=L \breve{I}+2 L_{g}\left(\breve{I}-\frac{U^{-1}+U}{2}\right), \\
& \beta=\frac{\breve{I}}{C}+\frac{1}{C_{g}}\left(\breve{I}-U^{-1}\right)(\breve{I}-U)=\frac{\breve{I}}{C}+\frac{2}{C_{g}}\left(\breve{I}-\frac{U^{-1}+U}{2}\right), \\
& \zeta=\rho \frac{U-U^{-1}}{2},
\end{aligned}
$$

where $\breve{I}$ is the identity operator (matrix). In particular, for the matrix entries we have

$$
\begin{aligned}
\alpha_{n, m} & =L \delta_{n, m}+L_{g}\left(2-\delta_{n-1, m}-\delta_{n+1, m}\right), \\
\beta_{n, m} & =\frac{1}{C} \delta_{n, m}+\frac{1}{C_{g}}\left(2-\delta_{n-1, m}-\delta_{n+1, m}\right), \\
\zeta_{n, m} & =\frac{\rho}{2}\left(\delta_{n-1, m}-\delta_{n+1, m}\right) .
\end{aligned}
$$

In view of (6.9) the representations (6.10) imply the following expressions for the Fourier transform of $\alpha, \beta$, and $\zeta$ :

$$
\begin{aligned}
\hat{\alpha}(k) & =L+2 L_{g}(1-\cos (k)), \\
\hat{\beta}(k) & =\frac{1}{C}+\frac{2}{C_{g}}(1-\cos (k)), \\
\hat{\zeta}(k) & =\rho \frac{e^{i k}-e^{-i k}}{2}=i \rho \sin (k), \\
\hat{\zeta}^{T}(k) & =-\hat{\zeta}(k)=-i \rho \sin (k) .
\end{aligned}
$$

In the general case of (5.18) we have

$$
\hat{D}_{H}(k)=\left[\begin{array}{ll}
\hat{\zeta}^{T}(k) \hat{\alpha}^{-1}(k) & -\hat{\zeta}^{T}(k) \hat{\alpha}^{-1}(k) \hat{\zeta}(k)-\hat{\beta}(k) \\
\hat{\alpha}^{-1}(k) & -\hat{\alpha}^{-1}(k) \hat{\zeta}(k)
\end{array}\right] .
$$

In the special case when $\hat{\alpha}(k), \hat{\beta}(k), \hat{\zeta}(k)$, and $\hat{\zeta}^{T}(k)=-\hat{\zeta}(k)$ are just scalar-valued functions of $k$, which evidently commute, the representation (6.13) takes a simpler form

$$
\hat{D}_{H}(k)=\left[\begin{array}{ll}
-\hat{\zeta}(k) \hat{\alpha}^{-1}(k) & \hat{\zeta}^{2}(k) \hat{\alpha}^{-1}(k)-\hat{\beta}(k) \\
\hat{\alpha}^{-1}(k) & -\hat{\zeta}(k) \hat{\alpha}^{-1}(k)
\end{array}\right] .
$$

Plugging the values (6.12) in (6.14) we obtain

$$
\hat{D}_{H}(k)=\left[\begin{array}{ll}
-i \hat{\alpha}^{-1}(k) \rho \sin (k) & -\hat{\alpha}^{-1}(k) \rho^{2} \sin ^{2}(k)-\hat{\beta}(k) \\
\hat{\alpha}^{-1}(k) & -i \hat{\alpha}^{-1}(k) \rho \sin (k)
\end{array}\right],
$$




$$
\hat{\alpha}(k)=L+2 L_{g}(1-\cos (k)), \hat{\beta}(k)=\frac{1}{C}+\frac{2}{C_{g}}(1-\cos (k)) .
$$

Equation (5.19) after the Fourier transform becomes

$$
\frac{d \hat{Q}(k, t)}{d t}=\hat{D}_{H}(k) \hat{Q}(k, t)
$$

where the $2 \times 2$ matrix $\hat{D}_{H}(k)$ is defined by (6.15). In particular, for time harmonic $\hat{Q}(k, t)=e^{i \omega t} \hat{Q}(k),(6.17)$ implies

$$
\left[\hat{D}_{H}(k)-i \omega\right] \hat{Q}(k)=0 .
$$

Therefore, the circuit characteristic equation is

$$
\operatorname{det}\left[\hat{D}_{H}(k)-i \omega\right]=0
$$

or, in view of (6.15), it takes the form (3.11).

The circuit equation (3.19) is just the Lagrangian equations (5.8) for the case (6.11).

6.2. Phase and group velocities. The inequalities (3.38) for the group velocities $u_{+}(\omega)$ and $u_{-}(\omega)$ are very important by many reasons. In particular, they show that even when spectral reciprocity is violated for any frequency $\omega$ in the spectrum $\left[\Omega_{-}, \Omega_{+}\right]$there will always be two wave packets of this frequency propagating in the opposite directions.

To verify the inequalities (3.38) we need to study in more detail the solutions $Z_{ \pm}(\omega)=e^{i K_{ \pm}(\omega)}$ to $(3.26)$ with coefficients defined by (3.22), (3.23). We will study those solution for complex frequencies of the form

$$
\omega^{\prime}=\omega-i \delta, \delta \geq 0
$$

Let us remark first of all that for all complex valued $\omega^{\prime}$ which are not in the interval $\left[\Omega_{-}, \Omega_{+}\right]$we have

$$
\left|Z_{ \pm}\left(\omega^{\prime}\right)\right| \neq 1 \text { for } \omega^{\prime} \text { in } C-\left[\Omega_{-}, \Omega_{+}\right]
$$

where $\mathbf{C}$ is the complex plane. Indeed, suppose that (6.21) is violated and, for instance, $Z_{+}\left(\omega^{\prime}\right)=e^{i k}$ for a real $k$, then plugging $z=e^{i k}$ in (3.26) gives

$$
b_{0}\left(\omega^{\prime}\right)+b_{1}\left(\omega^{\prime}\right) e^{-i k}+b_{1}\left(-\omega^{\prime}\right) e^{i k}=0 .
$$

If we view now the equality (6.22) as an equation for $\omega^{\prime}$ we find (6.22) is identical to (3.11) and, hence, $\omega^{\prime}=\Omega(k)$. This clearly contradicts the assumption that $\omega^{\prime}$ is not in the interval $\left[\Omega_{-}, \Omega_{+}\right]$. Hence the relation (6.21) is correct.

Let us set the indices \pm for $Z_{ \pm}\left(\omega^{\prime}\right)$ to provide the inequality

$$
\left|Z_{+}\left(\omega^{\prime}\right)\right| \geq\left|Z_{-}\left(\omega^{\prime}\right)\right|
$$

We want to show now that

$$
\left|Z_{+}\left(\omega^{\prime}\right)\right|>1,\left|Z_{-}\left(\omega^{\prime}\right)\right|<1 \text { for } \omega^{\prime} \text { in } \mathbf{C}-\left[\Omega_{-}, \Omega_{+}\right] .
$$


Note that

$$
Z_{ \pm}(\infty)=1+\frac{1}{2}\left[\frac{L}{L_{g}} \pm \sqrt{4+\frac{L^{2}}{L_{g}}}\right]
$$

and, in particular

$$
\left|Z_{+}(\infty)\right|>1,\left|Z_{-}(\infty)\right|<1
$$

Since $Z_{+}\left(\omega^{\prime}\right)$ and $Z_{-}\left(\omega^{\prime}\right)$ are continuous in $\omega^{\prime}$, then (6.26) together with (6.23) imply (6.24). Having in mind the representation (3.28) we can rewrite (6.24) as

$$
\begin{aligned}
\operatorname{Im} K_{+}\left(\omega^{\prime}\right)<0, & \operatorname{Im} K_{+}\left(\omega^{\prime}\right)>0 \\
& \text { for } \omega^{\prime} \text { in } \mathbf{C}-\left[\Omega_{-}, \Omega_{+}\right] .
\end{aligned}
$$

The inequalities (6.24) imply the following inequalities for the group velocities $u_{ \pm}(\omega)$ associated, respectively, with $Z_{ \pm}(\omega)$ and $K_{ \pm}(\omega)$ :

$$
u_{+}(\omega) \geq 0, u_{-}(\omega) \leq 0 \text { for } \omega \text { in }\left[\Omega_{-}, \Omega_{+}\right] .
$$

Indeed, for $\omega^{\prime}=\omega-i \delta$ where $\omega$ is in the spectrum $\left[\Omega_{-}, \Omega_{+}\right]$and $\delta>0$ is small we have

$$
\begin{aligned}
& Z_{ \pm}\left(\omega^{\prime}\right)=\exp \left\{i\left[K_{ \pm}(\omega)-K_{ \pm}^{\prime}(\omega) i \delta+O\left(\delta^{2}\right)\right]\right\} \\
= & Z_{ \pm}(\omega) \exp \left\{\left(1 / u_{ \pm}(\omega)\right) \delta+O\left(\delta^{2}\right)\right\} .
\end{aligned}
$$

These representations together with inequalities (6.24) imply the inequalities (6.28). Hence, for any frequently $\omega$ in the spectrum there are two group velocities one of which is nonnegative and another one is nonpositive.

Depending on the sign of the parameter $\rho$ one of the group velocities $u_{+}(\omega)$ and $-u_{-}(\omega)$ will always be greater. More precisely, for $\omega$ in the spectrum $\left[\Omega_{-}, \Omega_{+}\right]$we have

$$
\left\{\begin{array}{lll}
u_{+}(\omega) \leq-u_{-}(\omega) & \text { if } \quad \rho>0 \\
u_{+}(\omega) \geq-u_{-}(\omega) & \text { if } \quad \rho<0 .
\end{array}\right.
$$

To verify, we note that (3.26) implies

$$
Z_{+}\left(\omega^{\prime}\right) Z_{-}\left(\omega^{\prime}\right)=\frac{b_{1}\left(\omega^{\prime}\right)}{b_{1}\left(-\omega^{\prime}\right)} .
$$

We want to find now if the ration $\left|b_{1}\left(\omega^{\prime}\right) / b_{1}\left(-\omega^{\prime}\right)\right|$ is greater or smaller than 1 for $\delta>0$. From the explicit expression $(3.23)$ for $b_{1}(\omega)$ we obtain

$$
\left|b_{1}\left(\omega^{\prime}\right)\right|^{2}-\left|b_{1}\left(-\omega^{\prime}\right)\right|^{2}=4 \rho \delta\left(\left|\omega^{\prime}\right|^{2} L_{g}+\frac{1}{C_{g}}\right),
$$

which, in turn, implies

$$
\begin{cases}\left|b_{1}\left(\omega^{\prime}\right)\right|>\left|b_{1}\left(-\omega^{\prime}\right)\right| & \text { if } \quad \rho>0 \\ \left|b_{1}\left(\omega^{\prime}\right)\right|<\left|b_{1}\left(-\omega^{\prime}\right)\right| & \text { if } \quad \rho<0\end{cases}
$$

These inequalities together with (6.31) give

$$
\left\{\begin{array}{lll}
\ln \left|Z_{+}\left(\omega^{\prime}\right)\right|-\ln \left|Z_{-}\left(\omega^{\prime}\right)\right|^{-1}>0 & \text { if } \quad \rho>0 \\
\ln \left|Z_{+}\left(\omega^{\prime}\right)\right|-\ln \left|Z_{-}\left(\omega^{\prime}\right)\right|^{-1}<0 & \text { if } \quad \rho<0 .
\end{array}\right.
$$

The inequalities (6.34) and the representations (6.29) imply the inequalities (6.30). 
6.3. Wave numbers and the spectrum edges. The analytic expressions for the wave numbers $K_{ \pm}(\omega)$, defined by (3.28), can be found from (3.26). To analyze (3.26) we rewrite it using the following representation of the coefficients (3.22) and (3.23):

$$
\begin{aligned}
b_{1} & =\left|b_{1}\right| e^{-i \varkappa}, \\
\left|b_{1}\right| & =\sqrt{\left(L_{g} \omega^{2}-\frac{1}{C_{g}}\right)^{2}+(\rho \omega)^{2}}, \\
\varkappa & =\arctan \frac{\rho \omega}{L_{g} \omega^{2}-\frac{1}{C_{g}}} .
\end{aligned}
$$

Using these formulas we recast (3.26) as follows:

$$
\begin{aligned}
\zeta+\zeta^{-1} & =-\frac{b_{0}}{\left|b_{1}\right|}, \\
\zeta & =e^{-i \varkappa} z .
\end{aligned}
$$

In the case when the frequency $\omega$ falls in spectrum $\left[\Omega_{-}, \Omega_{+}\right]$we have $z=e^{i K}$ and, hence, $\zeta=e^{i k}$. This together with (6.38) yields

$$
2 \cos k=-\frac{b_{0}}{\left|b_{1}\right|}
$$

or

$$
k= \pm \arccos \left(-\frac{b_{0}}{2\left|b_{1}\right|}\right) .
$$

This implies the representation (3.39), (3.40), (3.41) for the wave numbers $K_{ \pm}(\omega)$. Observe now that from (3.39), (3.40), (3.41), and (3.42) it evidently follows that

$$
K_{-}(\omega) \geq K_{+}(\omega) .
$$

Note also that in view of $(3.29) K_{ \pm}(\omega)$ are inverse functions to the periodic function $\Omega(k)$ defined by (3.13). Note also that $\Omega(k)$ has exactly one minimum at a point $K_{\max }$ and exactly one maximum at point $K_{\min }$ on any of its periods. Let us choose the period in the form $\left[K_{\min }, K_{\min }+2 \pi\right]$ and

$$
K_{\min }<K_{\max }<K_{\min }+2 \pi
$$

where

$$
K_{\min }=K_{+}\left(\Omega_{-}\right) .
$$

Then $K_{-}(\omega)$ and $K_{+}(\omega)$ are, respectively, monotonically decreasing and increasing functions such that

$$
K_{-}\left(\Omega_{-}\right)<K_{-}\left(\Omega_{+}\right)=K_{+}\left(\Omega_{+}\right)<K_{+}\left(\Omega_{-}\right)=K_{-}\left(\Omega_{-}\right)+2 \pi .
$$

See the plots of wave vectors $K_{ \pm}(\omega)$ on Figures 3.9 and 3.10. The signs for $K_{ \pm}(\omega)$ in the formula (3.39) are chosen to match the signs of the derivatives $d K_{ \pm}(\omega) / d \omega$ which are related to the directions of the two group velocities (3.34). 
Observe also the shift $\varkappa$ associated with the spectral nonreciprocity depends only on the parameter $L_{g}, C_{g}$, and $\rho$ and does not depend on $L$ and $C$.

Band edges. Let us derive now the representations (3.15) for $\Omega_{ \pm}$. Note first that the frequency $\omega$ falls in spectrum $\left[\Omega_{-}, \Omega_{+}\right]$if and only if the solutions $z$ to (3.26) have the property $|z|=1$. As follows from (6.38) this can happen if and only if

$$
\left|\frac{b_{0}(\omega)}{b_{1}(\omega)}\right| \leq 2
$$

In particular, the band edges $\Omega_{-}$and $\Omega_{+}$can be found from the equation

$$
\left|\frac{b_{0}(\omega)}{b_{1}(\omega)}\right|=2
$$

or

$$
b_{0}^{2}(\omega)=4 b_{1}(\omega) b_{1}(-\omega)
$$

which, in view of (3.22) and (3.23), takes the form

$$
L a \omega^{4}-\left(a c+L b+4 \rho^{2}\right) \omega^{2}+c b=0,
$$

where

$$
a=\left(L+4 L_{g}\right), b=\frac{1}{C}+\frac{4}{C_{g}}, c=\frac{1}{C} .
$$

From (6.48) we immediately get the representations (3.15) for $\Omega_{ \pm}$.

6.4. Energy flow for a general lattice system. To describe the energy flow on a circuit we have first to split the total energy of the circuit between the sites of the lattice $\check{Z}$. This can be done in a few ways. For a circuit described by a Lagrangian function $\mathcal{L}$, such as (3.10), for instance, we will do it as follows. Let us break the Lagrangian function $\mathcal{L}$ into the sum of the form

$$
\mathcal{L}=\sum_{n} \ell_{n}, \ell_{n}=\ell_{n}\left(Y_{n}, \dot{Y}_{n}\right), Y_{n}=\left\{q_{n-1}, q_{n}, q_{n+1}\right\}
$$

We assume that it can be done. For the circuit (3.10) we will have (6.50) setting

$$
\ell_{n}\left(Y_{n}, \dot{Y}_{n}\right)=\ell_{n}^{(i)}+\ell_{n}^{(g i)}+\ell_{n}^{(k)}+\ell_{n}^{(p)}
$$

where

$$
\begin{gathered}
\ell_{n}^{(i)}=\frac{L \dot{Q}_{n}^{2}}{2}-\frac{Q_{n}^{2}}{2 C} \\
\ell_{n}^{(g)}=\frac{\rho}{4}\left(Q_{n-1} \dot{Q}_{n}-Q_{n} \dot{Q}_{n-1}\right)+\left(Q_{n} \dot{Q}_{n+1}-Q_{n+1} \dot{Q}_{n}\right) \\
\ell_{n}^{(k)}=\frac{L_{g}}{4}\left(\dot{Q}_{n}-\dot{Q}_{n-1}\right)^{2}+\left(\dot{Q}_{n+1}-\dot{Q}_{n}\right)^{2} ; \\
\ell_{n}^{(p)}=-\frac{1}{4 C_{g}}\left(Q_{n}-Q_{n-1}\right)^{2}+\left(Q_{n+1}-Q_{n}\right)^{2} .
\end{gathered}
$$


When making up the decomposition (6.51) we assigned to the site $n$, first, the terms of (3.10) which depends solely on the index $n$ and, second, one half of the terms which depend on either both $n$ and $n-1$ or $n$ and $n+1$. Having done that, we have split up evenly the terms depending on two adjacent indices between the sites associated with these indices. In (6.51) $\ell_{n}^{(i)}$ is the Lagrangian function of the elementary $L-C$ oscillator; the term $\ell_{n}^{(g i)}$ describes the coupling between the site $n$ and the adjacent sites $n-1$ and $n+1$ through the gyroscopic terms; the terms $\ell_{n}^{(k)}$ and $\ell_{n}^{(p)}$ describe the kinetic and potential coupling due to common inductances and capacitances for the site and the adjacent sites $n-1$ and $n+1$.

Assuming from now on the validity of the decomposition (6.51) we introduce the following representation of the Hamiltonian function:

$$
H=\sum_{n} E_{n}, E_{n}=p_{n} \dot{q}_{n}-\ell_{n}
$$

where

$$
\begin{aligned}
& p_{n}=\frac{\partial \mathcal{L}}{\partial \dot{q}_{n}}=\sum_{|s-n| \leq 1} \frac{\partial \ell_{s}}{\partial \dot{q}_{n}}, \\
& \dot{p}_{n}=\frac{\partial \mathcal{L}}{\partial q_{n}}=\sum_{|s-n| \leq 1} \frac{\partial \ell_{s}}{\partial q_{n}} .
\end{aligned}
$$

From (6.56) and (6.57) we get

$$
E_{n}=p_{n} \dot{q}_{n}-\ell_{n}=\left(\sum_{|s-n| \leq 1} \frac{\partial \ell_{s}}{\partial \dot{q}_{n}}\right) \dot{q}_{n}-\ell_{n} .
$$

This formula gives an expression of the instant energy of the system assigned to the site $n$. To get an expression of the energy flow $S_{n}$ we need to come up with the representation

$$
\frac{d}{d t} E_{n}=-\left(S_{n}-S_{n-1}\right)
$$

If (6.60) holds, $S_{n}$ can be interpreted as energy flow from the site $n$ to site $n+1$ and (6.60) the energy balance equation at the site $n$.

Using (6.59), (6.57), and (6.58) we obtain

$$
\frac{d}{d t} E_{n}=\dot{p}_{n} \dot{q}_{n}+p_{n} \ddot{q}_{n}-\sum_{|s-n| \leq 1} \frac{\partial \ell_{n}}{\partial q_{s}} \dot{q}_{s}-\sum_{|s-n| \leq 1} \frac{\partial \ell_{n}}{\partial \dot{q}_{s}} \ddot{q}_{s}
$$

or

$$
\frac{d}{d t} E_{n}=\sum_{s=n \pm 1}\left[\frac{\partial \ell_{s}}{\partial q_{n}} \dot{q}_{n}-\frac{\partial \ell_{n}}{\partial q_{s}} \dot{q}_{s}\right]+\sum_{s=n \pm 1}\left[\frac{\partial \ell_{s}}{\partial \dot{q}_{n}} \ddot{q}_{n}-\frac{\partial \ell_{n}}{\partial \dot{q}_{s}} \ddot{q}_{s}\right]
$$

Based on some general divergence-type representation (7.28), (7.29) described in the appendix we find the energy flow $S_{n}$ as follows. We introduce two matrix operators

$$
G_{n s}=-\frac{\partial \ell_{n}}{\partial q_{s}}, \dot{G}_{n s}=-\frac{\partial \ell_{n}}{\partial \dot{q}_{s}}
$$


Then

$$
\frac{d}{d t} E_{n}=[\widehat{G} \dot{q}]_{n}-[\widehat{\dot{G}} \ddot{q}]_{n}
$$

or

$$
\begin{aligned}
\frac{d}{d t} E_{n} & =-\left(S_{n}-S_{n-1}\right), \\
S_{n} & =-\left(G_{n, n+1} \dot{q}_{n+1}-G_{n+1, n} \dot{q}_{n}\right)-\left(\dot{G}_{n, n+1} \ddot{q}_{n+1}-\dot{G}_{n+1, n} \ddot{q}_{n}\right) .
\end{aligned}
$$

Combining now (6.65) with (6.62) we arrive at

$$
\begin{aligned}
S_{n}= & -\left(\frac{\partial \ell_{n+1}}{\partial q_{n}} \dot{q}_{n}-\frac{\partial \ell_{n}}{\partial q_{n+1}} \dot{q}_{n+1}\right) \\
& -\left(\frac{\partial \ell_{n+1}}{\partial \dot{q}_{n}} \ddot{q}_{n}-\frac{\partial \ell_{n}}{\partial \dot{q}_{n+1}} \ddot{q}_{n+1}\right) .
\end{aligned}
$$

6.5. Energy flow and energy velocity in a circuit. Using (6.51), (6.52)(6.55), (6.57), and (6.59) we consequently obtain the following representations for the site momentum and energy:

$$
\begin{gathered}
p_{n}=\frac{\rho}{2}\left(Q_{n-1}-Q_{n}\right)+\left(2 \dot{Q}_{n}-\dot{Q}_{n-1}-\dot{Q}_{n+1}\right), \\
E_{n}=E_{n ; \rho}+E_{n ; C_{g}}+E_{n ; L_{g}}+E_{n ; L C}, \\
E_{n ; \rho}=\frac{\rho\left[\left(\dot{Q}_{n-1}-\dot{Q}_{n+1}\right) Q_{n}+\left(Q_{n-1}-Q_{n}\right) \dot{Q}_{n}\right]}{4}, \\
E_{n ; L_{g}}=\frac{L_{g}\left[6 \dot{Q}_{n}^{2}-2\left(\dot{Q}_{n-1}+\dot{Q}_{n+1}\right) \dot{Q}_{n}-\dot{Q}_{n-1}^{2}-\dot{Q}_{n+1}^{2}\right]}{4}, \\
E_{n ; C_{g}}=\frac{\left(\dot{Q}_{n+1}-\dot{Q}_{n}\right)^{2}+\left(\dot{Q}_{n}-\dot{Q}_{n-1}\right)^{2}}{4 C_{g}}, \\
E_{n ; L C}=\frac{L}{2} \dot{Q}_{n}^{2}+\frac{1}{2 C} Q_{n}^{2} .
\end{gathered}
$$

Similarly using (6.66) we get the following representation for the site energy flow:

$$
\begin{aligned}
S_{n} & =S_{n ; \rho}+S_{n ; C_{g}}+S_{n ; L_{g}}, \\
S_{n ; \rho} & =\frac{\rho\left[\ddot{Q}_{n+1} Q_{n}+\ddot{Q}_{n} Q_{n+1}-2 \dot{Q}_{n} \dot{Q}_{n+1}\right]}{4}, \\
S_{n ; L_{g}} & =\frac{L_{g}\left(\ddot{Q}_{n+1}+\ddot{Q}_{n}\right)\left(\dot{Q}_{n}-\dot{Q}_{n+1}\right)}{2}, \\
S_{n ; C_{g}} & =\frac{\left(\dot{Q}_{n+1}+\dot{Q}_{n}\right)\left(Q_{n}-Q_{n+1}\right)}{2 C_{g}} .
\end{aligned}
$$

Consider now the case when $Q_{n}=\Psi_{n}(k)$ is the eigenmode of the form (3.30). Let $E(k)$ and $S(k)$ be, respectively, the time average of the instant energy (6.68) and energy flow (6.69) at any site $n$. From (6.68) and (6.69) one can derive the explicit representations (3.45), (3.46), (3.47), and (3.48) for the quantities $E(k)$ and $S(k)$ and the energy velocity $w(k)=S(k) / E(k)$. 
7. Appendix: Sequences of the divergence form. We say that a sequence $\left\{a_{n},-\infty<n<\infty\right\}$ is of the divergence form if there exists another sequence $b_{n}$ such that

$$
a_{n}=(D b)_{n}=b_{n}-b_{n-1},
$$

where

$$
D=\breve{I}-U,(U b)_{n}=b_{n-1} .
$$

In our applications the sequence $b_{n}$ can be interpreted as the flow.

If we treat the equality (7.1) as an equation for $b_{n}$ then, clearly, for any two solutions $b_{n}^{\prime}$ and $b_{n}^{\prime \prime}$ to (7.1) we must have

$$
b_{n}^{\prime}-b_{n}^{\prime \prime}=\text { const } .
$$

In addition to that, we can offer a solution of the form

$$
b_{n}=\sum_{m=n_{0}}^{n} a_{m},
$$

where $n_{0}$ is any fixed integer.

In situations of interest we need the representation (7.1) with an additional important requirement: $b_{n}$ must be a bounded sequence. Under that constraint (7.1) may have no solutions. From now on we will say that a sequence $a_{n}$ is of the divergent form if the representation (7.1) holds for a bounded sequence $b_{n}$.

Let us look at the divergence-type sequences of the form

$$
a_{n}=(\Gamma q)_{n},
$$

where $\Gamma=\left\{\Gamma_{n m},-\infty<n, m<\infty\right\}$ is a matrix. Any such a $\Gamma$ can be written as

$$
\Gamma=\sum_{p=-\infty}^{\infty} U^{p} \gamma^{(p)}
$$

for some diagonal matrices $\gamma^{(p)}$, i.e.,

$$
\left[\gamma^{(p)}\right]_{m n}=\gamma_{n}^{(p)} \delta_{n m},
$$

where $\delta_{n m}$ is the Kronecker symbol. For the cases of interest $\gamma^{(p)}$ are identically zero for large $|p|$ and, thus from now on we will assume that

$$
\gamma^{(p)}=0,|p| \geq R .
$$

Note that if $e_{n}$ is the standard basis in $l_{2}$, then

$$
U^{p} e_{n}=e_{n+p}
$$

and

$$
\Gamma_{n m}=\sum_{p=-\infty}^{\infty}\left(e_{n}, U^{p} \gamma^{(p)} e_{m}\right)=\sum_{p=-\infty}^{\infty} \gamma_{m}^{(p)} \delta_{n-p, m}=\gamma_{m}^{(n-m)}
$$




$$
\gamma_{m}^{(s)}=\Gamma_{m+s, m}
$$

If for a given matrix $\Gamma$ and arbitrary sequence $q_{n}$ the sequence $[\Gamma q]_{n}$ is of the divergence type then the operator $\Gamma$ must have the form

$$
\Gamma=(\breve{I}-U) \Phi,
$$

which implies the following divergence representation:

$$
[\Gamma q]_{n}=[\Phi q]_{n}-[\Phi q]_{n-1} .
$$

To find $\Phi$ let us write it in the form

$$
\Phi=\sum_{p=-\infty}^{\infty} U^{p} \phi^{(p)}
$$

Then

$$
\Gamma=(\breve{I}-U) \Phi=\sum_{p=-\infty}^{\infty} U^{p}\left(\phi^{(p)}-\phi^{(p-1)}\right),
$$

which, in view of (7.7), is equivalent to

$$
\gamma^{(p)}=\phi^{(p)}-\phi^{(p-1)} .
$$

Using this representation and (7.8) we find $\phi^{(p)}$,

$$
\phi^{(p)}=\sum_{s=-\infty}^{p} \gamma^{(s)}
$$

which together with (7.10) and (7.11) implies

$$
\begin{gathered}
\phi_{n}^{(p)}=\sum_{s=-\infty}^{p} \gamma_{n}^{(s)}=\sum_{s=-\infty}^{p} \Gamma_{n+s, n}=\sum_{s=-\infty}^{n+p} \Gamma_{s, n}, \\
\Phi_{n m}=\phi_{m}^{(n-m)}=\sum_{s=-\infty}^{n} \Gamma_{s, m} .
\end{gathered}
$$

Observe now that

$$
\phi^{(p)}=\left\{\begin{array}{lll}
0 & \text { if } & p \leq-R \\
\sum_{s=-\infty}^{\infty} \gamma^{(s)} & \text { if } \quad p \geq-R
\end{array}\right.
$$

If we want in the representation of $\Phi$ by (7.14) to have only a finite number of nonzero terms $\phi^{(p)}$ (to be quasi-local operator) then, in view of (7.20), we have to impose on the operator $\Gamma$ the following constraint:

$$
\sum_{s=-\infty}^{\infty} \gamma^{(s)}=0
$$


An arbitrary $\Gamma$ of the form (7.6) can be modified to satisfy (7.21), namely

$$
\widehat{\Gamma}=\Gamma-\sum_{s=-\infty}^{\infty} \gamma^{(s)} .
$$

Using (7.10) we get

$$
\begin{aligned}
\widehat{\Gamma}_{n m} & =\Gamma_{n m}-\sum_{s=-\infty}^{\infty} \gamma_{n}^{(s)} \delta_{n m}=\Gamma_{n m}-\sum_{s=-\infty}^{\infty} \Gamma_{m+s, m} \delta_{n m} \\
& =\Gamma_{n m}-\sum_{p=-\infty}^{\infty} \Gamma_{p m} \delta_{n m} .
\end{aligned}
$$

Applying now the formula (7.19) to the operator $\widehat{\Gamma}$ we get the following representation for the associated operator $\widehat{\Phi}$ :

$$
\begin{aligned}
\widehat{\Phi}_{n m} & =\sum_{s=-\infty}^{n} \widehat{\Gamma}_{s, m}=\sum_{s=-\infty}^{n}\left(\Gamma_{s m}-\sum_{p=-\infty}^{\infty} \Gamma_{p m} \delta_{s m}\right) \\
& =\sum_{s=-\infty}^{n} \Gamma_{s m}-\left(\sum_{p=-\infty}^{\infty} \Gamma_{p m}\right)\left(\sum_{s=-\infty}^{n} \delta_{s m}\right) \\
& =\left\{\begin{array}{lll}
-\sum_{s=n+1}^{\infty} \Gamma_{s m} & \text { if } & m \leq n, \\
\sum_{s=-\infty}^{n} \Gamma_{s m} & \text { if } & m>n .
\end{array}\right.
\end{aligned}
$$

In the special important case when

$$
\Gamma_{n m}=0 \text { if }|n-m|>1
$$

the formula (7.24) takes the form

$$
\widehat{\Phi}_{n m}=\left\{\begin{array}{lll}
0 & \text { if } & m<n, \\
-\Gamma_{n+1, n} & \text { if } & m=n, \\
\Gamma_{n, n+1} & \text { if } & m=n+1, \\
0 & \text { if } & m>n+1
\end{array}\right.
$$

or

$$
\widehat{\Phi}_{n m}=-\Gamma_{n+1, n} \delta_{n m}+\Gamma_{n, n+1} \delta_{n+1, m} .
$$

In particular, we have

$$
[\widehat{\Phi} q]_{n}=\Gamma_{n, n+1} q_{n+1}-\Gamma_{n+1, n} q_{n},
$$

and the following representation holds:

$$
[\widehat{\Gamma} q]_{n}=[\widehat{\Phi} q]_{n}-[\widehat{\Phi} q]_{n-1} .
$$




\section{REFERENCES}

[1] N. Ashcroft and N. Mermin, Solid State Physics, Holt, Rinehart and Winston, New York, London, 1976.

[2] L. Brillouin, Wave Propagation in Periodic Structures. Electric Filters and Crystal Lattices, 2nd ed., Dover, New York, 1953.

[3] S. L. Chuang, Physics of Optoelectric Devices, John Wiley \& Sons, New York, 1995.

[4] H. Carlin and A. Giordano, Network Theory, Prentice-Hall, Englewood Cliffs, NJ, 1964.

[5] L. Felsen and N. Marcuvits, Radiation and Scattering of Waves, Oxford University Press, IEEE Press, 1994.

[6] D. Greenwood, Classical Dynamics, Dover, New York, 1997.

[7] R. Harrington, Time-Harmonic Electromagnetic Fields, McGraw-Hill, New York, 1961.

[8] J. Helszajn, Microwave Engineering: Passive, Active and Nonreciprocal Circuits, McGrawHill, New York, 1992.

[9] J. D. JaCKson, Classical Electrodynamics, 2nd ed., John Wiley \& Sons, New York, London, Sydney, 1975.

[10] J. A. Kong, Electromagnetic Wave Theory, 2nd ed., John Wiley \& Sons, New York, 1990.

[11] P. Kuchment, Floquet Theory for Partial Differential Operators, Birkhäuser Verlag, Basel, 1993.

[12] M. Reed And B. Simon, Analysis of Operators, Vol. 4, Academic Press, New York, London, 1978.

[13] C. Lanczos, The Variational Principles of Mechanics, Dover, New York, 1970.

[14] L. PARs, A Treatise on Analytical Dynamics, Ox Bow Press, Woodbridge, CT, 1979.

[15] B. Tellegen, The gyrator, a new electric network element, Philips Research Reports, 3 (1948), pp. 81-101.

[16] I. Lifshits, M. Azbel, And M. Kaganov, Electron Theory of Metals, Consultants Bureau, New York, 1973

[17] A. Abrikosov, Fundamentals of the Theory of Metals, North-Holland, Amsterdam, 1988. 